\title{
Trial Review
}

COVID-19 studies are our top priority. For all other trials, there is a 4-week delay in processing a trial submitted/resubmitted to the ANZCTR and additional delays for updates of registered trials. We appreciate your patience.

The safety and scientific validity of this study is the responsibility of the study sponsor and investigators. Listing a study does not mean it has been endorsed by the ANZCTR. Before participating in a study, talk to your health care provider and refer to this information for consumers

\section{$<$ BACK}

\section{Trial registered on ANZCTR}

$\begin{array}{ll}\text { Registration number } & \text { (i) } \\ \text { Ethics application status } & \text { (i) Yes } \\ \text { Date submitted } & \text { (i) } 10 / 12 / 2014 \\ \text { Date registered } & \text { (i) } \\ \text { Date last updated } & \text { (i) }\end{array}$

Titles \& IDs

\section{Public title}

Scientific title

Secondary ID [1]

Universal Trial Number (UTN)

Trial acronym

Linked study record

\section{Health condition}

\section{Health condition(s) or problem(s) studied:}

Physical activity

Mental health

\section{Condition category}

Public Health

Mental Health

Intervention/exposure

\section{Study type}

Description of intervention(s) / exposure

Intervention code [1]

Intervention code [2]

Comparator / control treatment

Control group

\section{MOVE Frankston Study}

Randomised controlled trial of the impact of incentives and support to increase organised physical activity among inactive residents of Frankston

Nil

U1111-1165-0798

MOVE Frankston

Outcomes

Interventional

Incentives in the form of trial passes to the Peninsula Aquatic and Recreation Centre, with additional support through telephone contact, SMS reminders and written information about physical activity

Behaviour

Lifestyle

Control group members receive no treatment.

Active

\section{Condition code}

Health promotion/education

Studies of normal psychology, cognitive function and behaviour 


$\begin{array}{cl}\text { Primary outcome [1] } & \text { Physical activity participation } \\ \text { Timepoint [1] } & 12 \text { month and 24 months after recruitment } \\ \text { Primary outcome [2] } & \text { Mental well being } \\ \text { Timepoint [2] } & 12 \text { months and 24 months after recruitment } \\ \text { Secondary outcome [1] } & \text { Self efficacy } \\ \text { Timepoint [1] } & 12 \text { months and 24 months after recruitment } \\ \text { Secondary outcome [2] } & \text { Attitudes } \\ \text { Timepoint [2] } & 12 \text { months and 24 months after recruitment }\end{array}$

Eligibility

Key inclusion criteria

Minimum age

Maximum age

Gender

Can healthy volunteers participate?

Key exclusion criteria
Reside in Frankston City Council area; do not meet the Australian national physical activity guidelines; do not attend gym or leisure centre on a weekly basis

18 Years

70 Years

Both males and females

Yes

Inability to converse in English; physical illness or disability that prevents walking for 10 minutes or more: cognitive problem that impairs communication or reasoning

\section{Study design}

\section{Purpose of the study \\ Allocation to intervention \\ Procedure for enrolling a subject and allocating the treatment (allocation concealment procedures) \\ Methods used to generate the sequence in which subjects will be randomised (sequence generation) \\ Masking / blinding}

Who is / are masked / blinded?

\section{Prevention}

Randomised controlled trial

Recruitment and allocation were done by separate researchers. Allocation took place centrally several weeks after recruitment and baseline measurement by means of computer generated random numbers.

Simple randomisation using a randomised number sequence created by computer software

Blinded (masking used)

The people assessing the outcomes

Factorial

Intervention assignment

Other design features

Phase

Type of endpoint(s)

Statistical methods / analysis

\section{Phase 2}

Efficacy

Sample size was determined as the number needed to show a significant difference in weekly participation in organised physical activity. It is assumed that $10 \%$ of control subjects will become regular participants in organised physical activity. Therefore a sample size of 300 in each arm of the study will be required to show with 95\% confidence limits and $80 \%$ power: (i) a 10\% difference in outcome between participants in Intervention 1 and the control group (i.e., 20\% vs. 10\%); and (ii) a 10\% difference between those in Intervention 1 and Intervention 2 (i.e., 30\% vs. 20\%). To accommodate a $25 \%$ drop out among recruited participants over the two year course of the study, the target sample size was inflated to 400 per group.

\section{Recruitment}

Recruitment status

Closed: follow-up continuing

Date of first participant enrolment

Anticipated

Actual

$24 / 07 / 2014$

Date of last participant enrolment

Anticipated

Date of last data collection

Anticipated

Actual
Actual
$17 / 09 / 2014$ 
Target

Recruitment in Australia

Recruitment state(s) $\quad \mathrm{VIC}$

Recruitment postcode(s) [1] 3195 - Aspendale

Recruitment postcode(s) [2] 3196 - Chelsea

Recruitment postcode(s) [3] 3197 - Carrum

Recruitment postcode(s) [4] 3198 - Seaford

Recruitment postcode(s) [5] 3199 - Frankston

Recruitment postcode(s) [6] 3200 - Frankston North

Recruitment postcode(s) [7] 3201 - Carrum Downs

Recruitment postcode(s) [8] 3911 - Langwarrin South

Recruitment postcode(s) [9] 3910 - Langwarrin

Recruitment postcode(s) [10] 3977 - Cranbourne

Funding \& Sponsors

\begin{tabular}{ll} 
Funding source category [1] & Government body \\
Name [1] & Australian Research Council \\
Address [1] & GPO Box 2702 \\
& CANBERRA \\
& ACT 2601 \\
Country [1] & Australia \\
Funding source category [2] & Government body \\
Name [2] & Frankston City Council \\
Address [2] & 30 Davey Street, \\
& Frankston VIC 3199 \\
Country [2] & Australia \\
Primary sponsor type & University \\
Name & Monash University \\
Address & Research Office \\
& Monash University \\
& Building 3d \\
Country & Monash University \\
Secondary sponsor category [1] & Victoria 3800 \\
Name [1] & Australia \\
Address [1] & None \\
Country [1] & \\
\hline
\end{tabular}

Ethics approval

\section{Ethics application status}

Ethics committee name [1]

Ethics committee address [1]

\section{Ethics committee country [1]}

Date submitted for ethics approval [1]

Approval date [1]

Ethics approval number [1]
Yes

Monash University Human Reseach Ethics Committee

First Floor, Building $3 \mathrm{e}$

Room 111

Monash Research Office

Clayton Campus

Monash University VIC 3800

Australia

$26 / 05 / 2014$

CF14/1148 - 2014000497

Summary 
Trial website

Trial related presentations / publications

Public notes of physical activity. This study, conducted in partnership with Frankston City Council, will investigate the impact that establishing a major recreational and aquatic centre has upon physical activity and measures of well being in the community. It will test the additional impacts of using marketing strategies to boost centre usage, and thereby inform future action to multiply the health benefits of public investments in recreational infrastructure.

\section{Contacts}

\section{Principal investigator}

Name

Address

Country

Phone

Fax

Email

\section{A/Prof Ben Smith}

School of Public Health and Preventive Medicine

Monash University

Level 6

99 Commercial Rd

Melbourne, Victoria, 3004

Australia

+61399031654

ben.smith@monash.edu

Contact person for public queries

Name

Address

Country

Phone

Fax

Email
A/Prof Ben Smith

School of Public Health and Preventive Medicine Monash University

Level 6

99 Commercial Rd

Melbourne, Victoria, 3004

Australia

+61399031654

ben.smith@monash.edu

\section{Contact person for scientific queries}

Name

Address

Phone

Fax

Email

\section{A/Prof Ben Smith}

School of Public Health and Preventive Medicine Monash University

Level 6

99 Commercial Rd

Melbourne, Victoria, 3004

Australia

$+61399031654$

ben.smith@monash.edu

No information has been provided regarding IPD availability

\section{Summary results}

No Results

\section{$<$ BACK}

